\title{
The Weak Order on Pattern-Avoiding Permutations
}

\author{
Brian Drake \\ Department of Mathematics \\ Grand Valley State University \\ Allendale, MI, U.S.A. \\ drakebr@gvsu .edu
}

Submitted: Jan 1, 2014; Accepted: Jul 18, 2014; Published: Jul 25, 2014

Mathematics Subject Classifications: 05A05; 06A12; 06B99

\begin{abstract}
The weak order on the symmetric group is a well-known partial order which is also a lattice. We consider subposets of the weak order consisting of permutations avoiding a single pattern, characterizing the patterns for which the subposet is a lattice. These patterns have only a single small ascent or descent. We prove that all patterns for which the subposet is a sublattice have length at most three.
\end{abstract}

\section{Introduction}

For any positive integer $n$, the weak order on the symmetric group $S_{n}$ is well known to be a lattice [7], [13]. There are also some interesting subposets of the weak order which are lattices, some of which can be described using pattern avoidance. In particular, the Tamari lattice is isomorphic to the weak order on 312 avoiding permutations ([4], 9.6. Theorem), [9]. Additionally, the Boolean lattice is isomorphic to the weak order on 312 and 231 avoiding permutations ([8], Lemme 4). Both the Tamari lattice and Boolean lattice are actually sublattices of the weak order. The Bruhat order on pattern avoiding permutations has also been studied [1].

In this article we consider the case of avoiding a single pattern. Our main result is the following.

Theorem 1. $S_{n}(\tau)$ is a lattice for all $n$ if and only if

1. $\tau$ has exactly one descent, and its magnitude is at most two, or

2. $\tau$ has exactly one ascent, and its magnitude is at most two. 

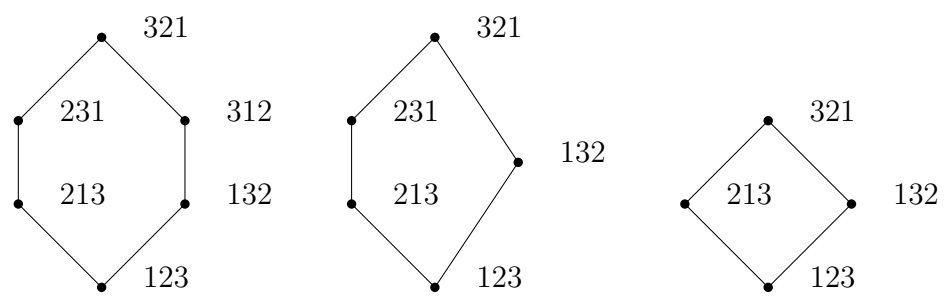

Figure 1: The weak order on $S_{3}, S_{3}(312)$, and $S_{3}(\{312,231\})$.

If we insist that the join and meet operations in our subposet agree with those in $S_{n}$, then it turns out that both (1) and (2) must be satisfied. These conditions are sufficient, and the result simplifies to the following.

Theorem 2. $S_{n}(\tau)$ is a sublattice of $S_{n}$ for all $n$ if and only if $\tau$ is one of 1, 12, 21, 213, 132, 231, or 312.

The weak order on permutations avoiding any one of 213, 132, 231, or 312 is isomorphic to the Tamari lattice. The others are either empty or a single vertex.

In the course of proving these theorems, we find a somewhat surprising result.

Theorem 3. If $\tau$ is a pattern of length $k \geqslant 2$, the following are equivalent:

1. $S_{n}(\tau)$ is a lattice for all $n$.

2. $S_{k+1}(\tau)$ is a lattice.

This paper is organized as follows. Section 2 contains some preliminaries about pattern avoidance, lattices, and the weak order. Section 3 contains the proofs of the main theorems. In Section 4, we give some results about $S_{n}(T)$, where $T$ contains multiple patterns.

\section{Preliminaries}

\subsection{Permutations}

A permutation of $n$ letters is a bijection $\pi:[n] \rightarrow[n]$, where $[n]$ denotes $\{1,2, \ldots, n\}$. The set of all permutations of $n$ letters is denoted $S_{n}$. We write our permutations in one line notation as $\pi=\pi(1) \pi(2) \cdots \pi(n)$. Note that $\pi^{-1}(i)$ gives the position of the letter $i$ in the word $\pi(1) \pi(2) \cdots \pi(n)$.

A descent in a permutation $\pi$ occurs in position $i$ if $\pi(i)>\pi(i+1)$. The magnitude of a descent in position $i$ is $\pi(i)-\pi(i+1)$. Ascents and their magnitudes are defined analogously. An inversion is a pair $(i, j)$ with $i<j$ and $\pi^{-1}(i)>\pi^{-1}(j)$. That is, in the one line notation for $\pi, i$ appears to the right of $j$. We define the inversion set $I(\pi)$ of $\pi$ to be the set of all inversions in $\pi$. For example, $\pi=24513$ has an ascent of magnitude 1 in position 2, ascents of magnitude 2 in positions 1 and 4 , and a descent of magnitude 4 in position 3. Its inversion set is $I(24513)=\{(1,2),(1,4),(1,5),(3,4),(3,5)\}$. 
If $\pi \in S_{m}$ and $\sigma \in S_{n}$, then $\pi \oplus \sigma$ denotes the permutation in $S_{m+n}$, where $\pi$ acts on $\{1,2, \ldots, m\}$, and $\sigma$ acts on $\{m+1, m+2, \ldots, m+n\}$. Similarly, $\pi \ominus \sigma$ denotes the permutation in $S_{m+n}$, where $\pi$ acts on $\{n+1, n+2, \ldots, n+m\}$, and $\sigma$ acts on $\{1,2, \ldots, n\}$. For example, $132 \oplus 21=13254$ and $132 \ominus 21=35421$.

\subsection{Pattern Avoidance}

Let $w$ be a word consisting of $k$ distinct integers. The standardization of $w$, denoted $s t(w)$, is obtained by replacing the smallest entry of $w$ with a 1 , the second smallest with a 2 , and so on. A permutation $\pi \in S_{n}$ is said to contain a pattern $\tau \in S_{k}$ if there is a subsequence $\pi^{\prime}$ of $\pi$ such that $s t\left(\pi^{\prime}\right)=\tau$. Otherwise, $\pi$ is said to avoid $\tau$. For example, the permutation $\pi=1423$ contains the pattern 132 twice, since $s t(142)=132$ and $s t(143)=132$. The permutation 2134 avoids 132 . For a set of patterns $T$, we will use $S_{n}(T)$ to denote the permutations of length $n$ which avoid all of the patterns in $T$. We will also use $S_{n}(T)$ to denote the set $S_{n}(T)$ together with its order relation induced from the weak order. For simplicity of notation, we omit the set brackets for a single pattern.

\subsection{Lattices}

We assume that the reader is familiar with the definitions of partially ordered sets (posets), covering relations, Hasse diagrams, and dual posets ([11], Chapter 3), or [2].

A lattice $L$ is a poset with the following property. For all $x, y \in L$, the set of upper bounds $\{z \in L \mid z \geqslant x, z \geqslant y\}$ has a unique minimal element, called the join of $x$ and $y$ and denoted $x \vee y$, and the set of lower bounds $\{z \in L \mid z \leqslant x, z \leqslant y\}$ has a unique maximal element, called the meet of $x$ and $y$ and denoted $x \wedge y$.

One useful lemma which follows directly from the definition of a lattice is the following.

Lemma 4. Let $L$ be a lattice, with $u, v, x, y, z \in L$. Then $u \vee v=z=x \wedge y$ if and only if $\{t \in L \mid u \leqslant t, v \leqslant t, t \leqslant x, t \leqslant y\}=\{z\}$.

A join semi-lattice $L$ is a poset where every pair of elements $x, y \in L$ has a join $x \vee y$. A meet semi-lattice $L$ is a poset where every pair of elements $x, y \in L$ has a meet $x \wedge y$. $L$ is a lattice if and only if it is both a join semi-lattice and a meet semi-lattice. We will make use of the following lemma.

Lemma 5. ([11], Proposition 3.3.1.) Let $P$ be a finite meet semi-lattice with a unique maximal element. Then $P$ is a lattice.

Given posets $Q$ and $P$, where the elements of $Q$ are a subset of the elements of $P$, we say that $Q$ is an induced subposet of $P$ if $x \leqslant y$ in $Q$ if and only if $x \leqslant y$ in $P$.

In this paper we will be interested in showing that certain induced subposets are lattices. We are not assuming that the join and meet operations are the same in the induced subposet as in the larger lattice. When these operations do coincide, we use a stronger term. An induced subposet $P$ of a lattice $L$ is a join sublattice if $x, y \in P$ implies $x \wedge y \in P$, where $\wedge$ is taken in $L$. An induced subposet $P$ of a lattice $L$ is a meet sublattice 
if $x, y \in P$ implies $x \vee y \in P$. $P$ is a sublattice of $L$ if $P$ is both a join sublattice and a meet sublattice.

Our main tool for investigating induced subposets is the following technical result.

Lemma 6. Let $L$ be a join semi-lattice and $P$ an induced subposet. Suppose that for all $v \in L$, the set $\left\{v^{\prime} \in P \mid v^{\prime} \leqslant v\right\}$ has a unique maximal element or is the empty set. Then $P$ is a join sublattice of $L$.

Proof. Let $x, y \in P$, and suppose $z=x \vee y$ in $L$. We want to show that $z \in P$. The set $\left\{z^{\prime} \in P \mid z^{\prime} \leqslant z\right\}$ is nonempty since it contains both $x$ and $y$, so by assumption it has a unique maximal element $z^{*}$. The element $z^{*}$ is in $\left\{z^{\prime} \in P \mid z^{\prime} \leqslant z\right\}$, so we have $z^{*} \leqslant z$. By the maximality of $z^{*}$, we have $x \leqslant z^{*}$ and $y \leqslant z^{*}$. But since $z=x \vee y$, we also have $z \leqslant z^{*}$ by the definition of join. Hence $z=z^{*}$ so $z \in P$.

\subsection{Weak Order}

For permutations $\sigma, \pi \in S_{n}$, we define $\sigma \leqslant \pi$ if and only if $I(\sigma) \subseteq I(\pi)$. It follows from the definition that $\pi$ covers $\sigma$ if and only if $\pi$ is obtained from $\sigma$ by an adjacent transposition which changes an ascent to a descent. Figure 1 includes the Hasse diagram of the weak order on $S_{3}$. The weak order on $S_{n}$ is well known to be a lattice [7], [13]. Throughout this paper, we will abuse notation and let $S_{n}$ denote the set of permutations of length $n$ together with the order relation given by the weak order.

There are some standard bijections of the weak order which will be useful. Let $w_{0} \in S_{n}$ be the strictly decreasing permutation $n(n-1) \cdots 21$.

Lemma 7. ([3], Proposition 3.1.5.) Let $\pi \in S_{n}$.

(i) $\pi \mapsto \pi w_{0}$ and $\pi \mapsto w_{0} \pi$ are order reversing bijections of the weak order on $S_{n}$.

(ii) $\pi \mapsto w_{0} \pi w_{0}$ is an order preserving bijection of the weak order on $S_{n}$.

The permutation $\pi w_{0}$ reverses the positions in the word $\pi$ and $w_{0} \pi$ reverses the values of the entries, switching $i$ and $(n+1-i)$, while $w_{0} \pi w_{0}$ does both commuting operations. For example, if $\pi=14235$, then $\pi w_{0}=53241, w_{0} \pi=52431$, and $w_{0} \pi w_{0}=13425$.

It follows immediately that $\pi$ avoids $\tau$ if and only if $\pi w_{0}$ avoids $\tau w_{0}$. The same is true for the other two bijections. Combined with Lemma 7, we get the following.

Lemma 8. Let $\pi \in S_{n}(T)$.

(i) $\pi \mapsto \pi w_{0}$ and $\pi \mapsto w_{0} \pi$ are order reversing bijections of the weak order on $S_{n}(T)$.

(ii) $\pi \mapsto w_{0} \pi w_{0}$ is an order preserving bijection of the weak order on $S_{n}(T)$. 


\section{Proof of Theorems 1,2 and 3}

\subsection{Terminology}

Let us introduce some terminology to be used in this section. Given a poset $P$ and elements $\sigma, \sigma^{\prime}, \mu, \mu^{\prime} \in P$ such that $\mu$ covers both $\sigma$ and $\sigma^{\prime}$ and $\mu^{\prime}$ covers both $\sigma$ and $\sigma^{\prime}$, we say that $\sigma, \sigma^{\prime}, \mu$, and $\mu^{\prime}$ form a butterfly in the Hasse diagram of $P$, and that $P$ contains a butterfly in its Hasse diagram. We call $\mu$ and $\mu^{\prime}$ the top elements of the butterfly and $\sigma$ and $\sigma^{\prime}$ the bottom elements of the butterfly. See Figure 2.

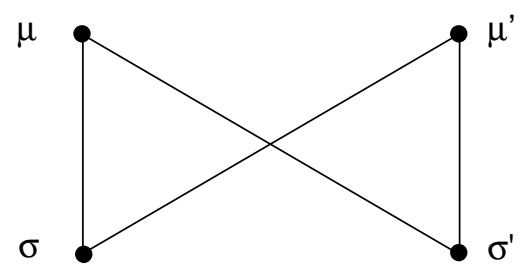

Figure 2: A butterfly in a Hasse diagram.

Note that any poset $P$ which contains a butterfly in its Hasse diagram cannot be a lattice or semi-lattice, since the meet of the top elements and the join of the bottom elements of the butterfly do not exist.

Lemma 9. If the Hasse diagram of $S_{n}(\tau)$ contains a butterfly, then so does the Hasse diagram of $S_{m}(\tau)$ for $m \geqslant n$.

Proof. Suppose $\sigma, \sigma^{\prime}, \mu$, and $\mu^{\prime}$ form a butterfly in the Hasse diagram of $S_{n}(\tau)$. Suppose also that $\tau$ ends with a descent in position $k-1$. In this case, define

$$
\begin{aligned}
\sigma_{m} & =\sigma \oplus 12 \cdots(m-n) \\
\sigma_{m}^{\prime} & =\sigma^{\prime} \oplus 12 \cdots(m-n) \\
\mu_{m} & =\mu \oplus 12 \cdots(m-n) \\
\mu_{m}^{\prime} & =\mu^{\prime} \oplus 12 \cdots(m-n) .
\end{aligned}
$$

Let us show that $\sigma_{m}, \sigma_{m}^{\prime}, \mu_{m}$, and $\mu_{m}^{\prime}$ avoid $\tau$. We know that $\tau$ ends with a descent and $\sigma_{m}, \sigma_{m}^{\prime}, \mu_{m}$, and $\mu_{m}^{\prime}$ each end with an increasing sequence of their greatest $(m-n)$ letters. So if any of these contains $\tau$, the occurrence of $\tau$ would have to be in the first $n$ letters. But these letters are one of $\sigma, \sigma^{\prime}, \mu$, or $\mu^{\prime}$, each of which avoids $\tau$. Hence $\sigma_{m}, \sigma_{m}^{\prime}, \mu_{m}, \mu_{m}^{\prime} \in S_{m}(\tau)$.

Notice that the inversion sets are unchanged: $I\left(\sigma_{m}\right)=I(\sigma), I\left(\sigma_{m}^{\prime}\right)=I\left(\sigma^{\prime}\right), I\left(\mu_{m}\right)=$ $I(\mu)$, and $I\left(\mu_{m}^{\prime}\right)=I\left(\mu^{\prime}\right)$. Therefore we have $\sigma_{m} \leqslant \mu_{m}, \sigma_{m} \leqslant \mu_{m}^{\prime}, \sigma_{m}^{\prime} \leqslant \mu_{m}, \sigma_{m}^{\prime} \leqslant \mu_{m}^{\prime}$, and these relations are covering relations. That is, $\sigma_{m}, \sigma_{m}^{\prime}, \mu_{m}$, and $\mu_{m}^{\prime}$ form a butterfly in the Hasse diagram of $S_{m}(\tau)$. 
For the remaining case, suppose that $\tau$ ends with an ascent. Define

$$
\begin{aligned}
\sigma_{m} & =\sigma \ominus(m-n) \cdots 21 \\
\sigma_{m}^{\prime} & =\sigma^{\prime} \ominus(m-n) \cdots 21 \\
\mu_{m} & =\mu \ominus(m-n) \cdots 21 \\
\mu_{m}^{\prime} & =\mu^{\prime} \ominus(m-n) \cdots 21 .
\end{aligned}
$$

An analogous argument shows that $\mu_{m}, \mu_{m}^{\prime}, \sigma_{m}$ and $\sigma_{m}^{\prime}$ form a butterfly in the Hasse diagram of $S_{m}(\tau)$.

\subsection{Proofs}

For the rest of this section, let $\tau$ be a pattern of length $k$, with $k \geqslant 2$. We prove our main results using three intermediate propositions.

Proposition 10. Suppose $\tau$ has at least two ascents and at least two descents. Then $S_{n}(\tau)$ is not a meet semi-lattice or a join semi-lattice for $n \geqslant k$.

Proof. Choose integers $a_{1}, a_{2}, d_{1}, d_{2}$ such that ascents of $\tau$ occur in positions $a_{1}$ and $a_{2}$ and descents of $\tau$ occur in positions $d_{1}$ and $d_{2}$. That is, we have $\tau\left(a_{1}\right)<\tau\left(a_{1}+1\right), \tau\left(a_{2}\right)<$ $\tau\left(a_{2}+1\right), \tau\left(d_{1}\right)>\tau\left(d_{1}+1\right)$, and $\tau\left(d_{2}\right)>\tau\left(d_{2}+1\right)$.

By Lemma 9 , it suffices to find a butterfly in the Hasse diagram of $S_{k}(\tau)$. Define permutations

$$
\begin{aligned}
& \mu_{1}=\tau(1) \tau(2) \cdots \tau\left(a_{1}-1\right) \tau\left(a_{1}+1\right) \tau\left(a_{1}\right) \tau\left(a_{1}+2\right) \cdots \tau(k) \\
& \mu_{2}=\tau(1) \tau(2) \cdots \tau\left(a_{2}-1\right) \tau\left(a_{2}+1\right) \tau\left(a_{2}\right) \tau\left(a_{2}+2\right) \cdots \tau(k) \\
& \sigma_{1}=\tau(1) \tau(2) \cdots \tau\left(d_{1}-1\right) \tau\left(d_{1}+1\right) \tau\left(d_{1}\right) \tau\left(d_{1}+2\right) \cdots \tau(k) \\
& \sigma_{2}=\tau(1) \tau(2) \cdots \tau\left(d_{2}-1\right) \tau\left(d_{2}+1\right) \tau\left(d_{2}\right) \tau\left(d_{2}+2\right) \cdots \tau(k) .
\end{aligned}
$$

Notice that $\mu_{i}$ is obtained from $\tau$ by changing the ascent in position $a_{i}$ to an descent, and that $\sigma_{i}$ is obtained from $\tau$ by changing the descent in position $d_{i}$ to an ascent. We have

$$
\begin{aligned}
& I\left(\mu_{1}\right)=I(\tau) \cup\left\{\left(\tau\left(a_{1}\right), \tau\left(a_{1}+1\right)\right)\right\} \\
& I\left(\mu_{2}\right)=I(\tau) \cup\left\{\left(\tau\left(a_{2}\right), \tau\left(a_{2}+1\right)\right)\right\} \\
& I\left(\sigma_{1}\right)=I(\tau) \backslash\left\{\left(\tau\left(d_{1}+1\right), \tau\left(d_{1}\right)\right)\right\} \\
& I\left(\sigma_{2}\right)=I(\tau) \backslash\left\{\left(\tau\left(d_{2}+1\right), \tau\left(d_{2}\right)\right)\right\} .
\end{aligned}
$$

By our definition of the weak order on $S_{k}$ in terms of inversion sets, we have $\mu_{1} \wedge \mu_{2}=\tau=$ $\sigma_{1} \vee \sigma_{2}$, where the meet and join are taken in $S_{k}$. By Lemma $4, \tau$ is the only permutation in $S_{k}$ between $\mu_{1}, \mu_{2}, \sigma_{1}$ and $\sigma_{2}$. Recall that $\tau$ has length $k$. A permutation $\pi$ of length $k$ contains a pattern $\tau$ of length $k$ if and only if $\pi=\tau$, so $S_{k}(\tau)=S_{k} \backslash\{\tau\}$ as sets. Then by the definition of an induced subposet, $\mu_{1}, \mu_{2}, \sigma_{1}$ and $\sigma_{2}$ form a butterfly in the Hasse diagram of $S_{k}(\tau)$. 
Proposition 11. Suppose $\tau$ has exactly one descent, and its magnitude is at least 3. Then $S_{n}(\tau)$ is not a meet semi-lattice or a join semi-lattice for $n \geqslant k+1$.

Proof. Given such a pattern $\tau$, we have a set of letters $\{a, b, c, d\}$ which appear in $\tau$, with $a<b<c<d$ in the usual integer ordering, and $a$ appearing immediately to the right of $d$ in $\tau$. That is, $\tau$ has a descent in position $\pi^{-1}(d)$ of magnitude $d-a \geqslant 3$. Since $\tau$ has exactly one descent, it can be decomposed into two increasing subwords of consecutive letters. One is $\tau(1)<\tau(2)<\cdots d$ and the other is $a<\cdots<\tau(k)$. Therefore we can assume that $a, b, c$, and $d$ appear consecutively in $\tau$. There are four possibilities for $\tau$ :

(i) $\tau=\tau_{\text {init }} b c d a \tau_{\text {end }}$

(ii) $\tau=\tau_{\text {init }} b d a c \tau_{\text {end }}$

(iii) $\tau=\tau_{\text {init }} c d a b \tau_{\text {end }}$

(iv) $\tau=\tau_{\text {init }} d a b c \tau_{\text {end }}$

where $\tau_{\text {init }}$ and $\tau_{\text {end }}$ are the appropriate increasing subwords. In each case we want to find a butterfly in the Hasse diagram of $S_{k+1}(\tau)$, which completes the proof by Lemma 9 .

Case $(i)$. Choose $\tau^{\prime} \in S_{k+1}$ such that

$$
\tau^{\prime}=\tau_{\text {init }}^{\prime} b(c+1)(d+1) s a \tau_{\text {end }}^{\prime}
$$

with $a<b<s<(c+1)<(d+1)$. Define

$$
\begin{aligned}
\mu_{1} & =\tau_{\text {init }}^{\prime}(c+1) b(d+1) s a \tau_{\text {end }}^{\prime} \\
\mu_{2} & =\tau_{\text {init }}^{\prime} b(d+1)(c+1) s a \tau_{\text {end }}^{\prime} \\
\sigma_{1} & =\tau_{\text {init }}^{\prime} b(c+1) a(d+1) s \tau_{\text {end }}^{\prime} \\
\sigma_{2} & =\tau_{\text {init }}^{\prime} b(c+1) s a(d+1) \tau_{\text {end }}^{\prime} .
\end{aligned}
$$

First, we want to show that $\mu_{1}, \mu_{2}, \sigma_{1}$, and $\sigma_{2}$ avoid $\tau$. Since $\tau$ has length $k$, an occurrence of $\tau$ in one of these permutations would use all letters but one. Such an occurrence would start with an increasing sequence of $\left|\tau_{\text {init }}\right|+3$ letters, followed by a descent. This is impossible in $\mu_{1}, \mu_{2}$ and $\sigma_{2}$. The letter after the descent in an occurrence of $\tau$ must be smaller than the previous three. This is impossible in $\sigma_{1}$. Hence $\mu_{1}, \mu_{2}, \sigma_{1}, \sigma_{2} \in S_{k+1}(\tau)$.

Next we want to verify that $\sigma_{1} \leqslant \mu_{1}, \sigma_{1} \leqslant \mu_{2}, \sigma_{2} \leqslant \mu_{1}$, and $\sigma_{2} \leqslant \mu_{2}$. This is immediate from the inversion sets

$$
\begin{aligned}
& I\left(\mu_{1}\right)=I\left(\tau^{\prime}\right) \cup\{(b,(c+1))\} \\
& I\left(\mu_{2}\right)=I\left(\tau^{\prime}\right) \cup\{((c+1),(d+1))\} \\
& I\left(\sigma_{1}\right)=I\left(\tau^{\prime}\right) \backslash\{(a, s),(a,(d+1))\} \\
& I\left(\sigma_{2}\right)=I\left(\tau^{\prime}\right) \backslash\{(a,(d+1)),(s,(d+1))\} .
\end{aligned}
$$

We want to show that all permutations in the set $\left\{\xi \in S_{k+1} \mid \xi \leqslant \mu_{1}, \xi \leqslant \mu_{2}, \sigma_{1} \leqslant\right.$ $\left.\xi, \sigma_{2} \leqslant \xi\right\}$ contain the pattern $\tau$. Since $\mu_{1}$ and $\mu_{2}$ cover $\tau^{\prime}$, any such permutation $\xi$ must 
satisfy $\xi \leqslant \tau^{\prime}$. The possible inversion sets are $I\left(\tau^{\prime}\right), I\left(\tau^{\prime}\right) \backslash\{(a, s)\}, I\left(\tau^{\prime}\right) \backslash\{(s,(d+1))\}$, and $I\left(\tau^{\prime}\right) \backslash\{(a,(d+1))\}$. The first is the inversion set for $\tau^{\prime}$, which contains $\tau$. The second is the inversion set for $\xi_{1}=\tau_{\text {init }}^{\prime} b(c+1)(d+1) a s \tau_{\text {end }}$, which contains $\tau$ by removing $s$. The third is the inversion set for $\xi_{2}=\tau_{\text {init }}^{\prime} b(c+1) s(d+1) a \tau_{\text {end }}$, which contains $\tau$ by removing $s$. The fourth is not the inversion set of a permutation, since to move $a$ left of $(d+1)$, either $a$ must move left of $s$ or $s$ must move left of $(d+1)$, removing another inversion. By the definition of an induced subposet, we have shown that $\mu_{1}, \mu_{2}, \sigma_{1}$, and $\sigma_{2}$ form a butterfly in the Hasse diagram of $S_{k+1}(\tau)$.

Case (ii). In this case we have $\tau=\tau_{\text {init }} b d a c \tau_{\text {end }}$ with $a<b<c<d$. Choose $\tau^{\prime} \in S_{k+1}$ by

$$
\tau=\tau_{\text {init }}^{\prime} b(d+1) s a(c+1) \tau_{\text {end }}^{\prime}
$$

with $a<b<s<(c+1)<(d+1)$. Define the following permutations:

$$
\begin{aligned}
\mu_{1} & =\tau_{\text {init }}^{\prime}(d+1) b s a(c+1) \tau_{\text {end }}^{\prime} \\
\mu_{2} & =\tau_{\text {init }}^{\prime} b(d+1) s(c+1) a \tau_{\text {end }}^{\prime} \\
\sigma_{1} & =\tau_{\text {init }}^{\prime} b a(d+1) s(c+1) \tau_{\text {end }}^{\prime} \\
\sigma_{2} & =\tau_{\text {init }}^{\prime} b s a(d+1)(c+1) \tau_{\text {end }}^{\prime} .
\end{aligned}
$$

We leave to the reader the argument that $\mu_{1}, \mu_{2}, \sigma_{1}$, and $\sigma_{2}$ form a butterfly in the Hasse diagram of $S_{k+1}(\tau)$. It is entirely analogous to Case $(i)$.

Case (iii). We have $\tau=\tau_{\text {init }} c d a b \tau_{\text {end }}$ with $a<b<c<d$. This is the same as in Case (ii), except $b$ and $c$ are switched. In Case $(i i)$ we did not need to use the relation $b<c$, so the same argument works in this case.

Case $(i v)$. This case is equivalent to Case $(i)$ by using the symmetry operation $\pi \mapsto w_{0} \pi w_{0}$ described in Lemma 8.

Proposition 12. Suppose $\tau$ has exactly one descent, and its magnitude is at most two. Then $S_{n}(\tau)$ is a join sublattice of $S_{n}$.

Proof. Let $\pi$ be a permutation which contains $\tau$. Choose an occurrence of $\tau$ in positions $i_{1}, i_{2}, \ldots, i_{k}$ with the descent in position $i_{h}$, such that $i_{h+1}-i_{h}$ is minimal.

First, we want to show that $\pi\left(i_{h}+1\right), \pi\left(i_{h}+2\right), \ldots, \pi\left(i_{h+1}-1\right)$ are each less than $\pi\left(i_{h+1}\right)$ or greater than $\pi\left(i_{h}\right)$. Suppose there is such a $\pi(j)$ with $\pi\left(i_{h+1}\right)<\pi(j)<\pi\left(i_{h}\right)$ and $i_{h}<j<i_{h+1}$. Then either

$$
\begin{gathered}
\text { st }\left(\pi\left(i_{1}\right) \pi\left(i_{2}\right) \cdots \pi\left(i_{h-1}\right) \pi(j) \pi\left(i_{h+1}\right) \cdots \pi\left(i_{k}\right)\right)=\tau, \text { or } \\
\text { st }\left(\pi\left(i_{1}\right) \pi\left(i_{2}\right) \cdots \pi\left(i_{h}\right) \pi(j) \pi\left(i_{h+2}\right) \cdots \pi\left(i_{k}\right)\right)=\tau
\end{gathered}
$$

depending on the relative sizes of $\pi\left(i_{h-1}\right), \pi\left(i_{h}\right), \pi(j), \pi\left(i_{h+1}\right)$, and $\pi\left(i_{h+2}\right)$. Here we are using the fact that $\tau$ has exactly one descent, which is of magnitude at most 2 . So we have another occurrence of $\tau$ with the letters forming the descent appearing closer together in $\pi$, contradicting the minimality of $i_{h+1}-i_{h}$. Hence $\pi\left(i_{h}+1\right), \pi\left(i_{h}+2\right), \ldots, \pi\left(i_{h+1}-1\right)$ are each less than $\pi\left(i_{h+1}\right)$ or greater than $\pi\left(i_{h}\right)$. 
In other words, $\left\{\pi(j) \mid i_{h}<j<i_{h+1}\right\}=X \cup Y$, where

$$
\begin{aligned}
& X=\left\{\pi(j) \mid i_{h}<j<i_{h+1}, \pi(j)<\pi\left(i_{h+1}\right)\right\} \\
& Y=\left\{\pi(j) \mid i_{h}<j<i_{h+1}, \pi\left(i_{h}\right)<\pi(j)\right\} .
\end{aligned}
$$

Let $x_{1}, x_{2}, \ldots, x_{r}$ be the elements of $X$, ordered such that if $p<q$, then $x_{p}$ appears to the left of $x_{q}$ in $\pi$. Let $y_{1}, y_{2}, \ldots, y_{t}$ be the elements of $Y$ ordered in the same fashion.

Now let $\pi^{\prime} \in S_{n}(\tau)$ with $\pi^{\prime}<\pi$. We want to show that $\pi^{\prime} \leqslant \pi_{0}$, where

$$
\pi_{0}=\pi(1) \pi(2) \cdots \pi\left(i_{h}-1\right) x_{1} x_{2} \cdots x_{r} \pi\left(i_{h+1}\right) \pi\left(i_{h}\right) y_{1} y_{2} \cdots y_{t} \pi\left(i_{h+1}+1\right) \cdots \pi(n) .
$$

Since $\pi\left(i_{1}\right), \pi\left(i_{2}\right), \ldots, \pi\left(i_{h}\right)$ form an increasing subsequence in $\pi$, these letters must also appear in increasing order from left to right in $\pi^{\prime}$, else $I\left(\pi^{\prime}\right) \nsubseteq I(\pi)$. Similarly, $\pi\left(i_{h+1}\right), \pi\left(i_{h+2}\right), \ldots, \pi\left(i_{k}\right)$ appear in increasing order from left to right in $\pi^{\prime}$. The subsequence $\pi\left(i_{1}\right), \pi\left(i_{2}\right), \ldots, \pi\left(i_{k}\right)$ does not form an occurrence of $\tau$ in $\pi^{\prime}$, so by the above $\pi\left(i_{h+1}\right)$ appears to the left of $\pi\left(i_{h}\right)$ in $\pi^{\prime}$. But $\pi_{0}$ is maximal among permutations less than $\pi$ with $\pi\left(i_{h+1}\right)$ appearing to the left of $\pi\left(i_{h}\right)$. Therefore $\pi^{\prime} \leqslant \pi_{0}$.

It is possible that $\pi_{0}$ contains $\tau$, but $\pi_{0}$ does not have an occurrence of $\tau$ with $\pi\left(i_{h+1}\right)$ and $\pi\left(i_{h}\right)$ forming the descent. Since $\pi$ has only finitely many letters, we can repeat this process and eventually end at a permutation $\pi^{*}$ such that $\pi^{*}$ avoids $\tau$, and every permutation $\pi^{\prime}$ which avoids $\tau$ and satisfies $\pi^{\prime}<\pi$ also satisfies $\pi^{\prime} \leqslant \pi^{*}$.

Now we can apply Lemma 6 to complete the proof.

We are now in a position to prove our main results. In fact, we have results about semi-lattices as well.

Theorem 13. $S_{n}(\tau)$ is a join sublattice of $S_{n}$ for all $n$ if and only if

1. $\tau$ has exactly one descent, and its magnitude is at most two, or

2. $\tau$ has no descents.

Proof. Let $\tau$ be a pattern of length $k$. Proposition 12 shows that if $\tau$ satisfies condition (1), then $S_{n}(\tau)$ is a join sublattice of $S_{n}$. Suppose that $\tau$ satisfies condition (2), so that $\tau=12 \cdots k$. In this case $\pi$ avoids $\tau$ if and only if $\pi$ does not contain an increasing subsequence of $k$ letters. If $\pi$ does not contain an increasing subsequence of $k$ letters and $\pi^{\prime} \geqslant \pi$, then $\pi^{\prime}$ does not contain an increasing subsequence of $k$ letters since $I(\pi) \subseteq I\left(\pi^{\prime}\right)$. Therefore $\pi, \sigma \in S_{n}(\tau)$ implies $\pi \vee \sigma \in S_{n}(\tau)$. That is, $S_{n}(\tau)$ is a join sublattice.

Now we want to show conditions (1) and (2) are necessary for $S_{n}(\tau)$ to be a join sublattice of $S_{n}$. Propositions 10 and 11 rule out all cases, except when $\tau$ has exactly one ascent, that ascent has magnitude at most 2, and $\tau$ has at least two descents. Suppose $\tau$ is of this type. Then $\tau$ is the join of two permutations, each obtained by changing one of the descents to an ascent. But these two permutations are in $S_{k}(\tau)$, so it cannot be a join sublattice.

Theorem 14. $S_{n}(\tau)$ is a join semi-lattice for all $n$ if and only if 
1. $\tau$ has exactly one descent, and its magnitude is at most two, or

2. $\tau$ has exactly one ascent, and its magnitude is at most two, or

3. $\tau$ has no descents.

Proof. Propositions 10 and 11 show that conditions (1), (2), and (3) are necessary. By Theorem 13, conditions (1) and (3) are sufficient. We want to show that condition (2) is as well.

Suppose $\tau$ has exactly one ascent, which is of magnitude at most two. Then by using $\pi \mapsto \pi w_{0}$ of Lemma 8 and Proposition 12, the dual poset to $S_{n}(\tau)$ is a join semi-lattice. That is, $S_{n}(\tau)$ is a meet semi-lattice. Since $n(n-1) \cdots 21$ avoids $\tau, S_{n}(\tau)$ has a maximal element. By the dual to Lemma $5, S_{n}(\tau)$ is a lattice, and hence a join semi-lattice.

Using the map $\pi \mapsto \pi w_{0}$ of Lemma 8, we have dual results.

Theorem 15. $S_{n}(\tau)$ is a meet sublattice of $S_{n}$ for all $n$ if and only if

1. $\tau$ has exactly one ascent, and its magnitude is at most two, or

2. $\tau$ has no ascents.

Theorem 16. $S_{n}(\tau)$ is a meet semi-lattice for all $n$ if and only if

1. $\tau$ has exactly one ascent, and its magnitude is at most two, or

2. $\tau$ has exactly one descent, and its magnitude is at most two, or

3. $\tau$ has no ascents.

Since $S_{n}(\tau)$ is a lattice if and only if it is both a meet semi-lattice and a join semilattice, we have proven Theorem 1 . We have also shown that the only $\tau$ for which $S_{n}(\tau)$ is a semi-lattice but not a lattice are the strictly increasing and strictly decreasing patterns.

We can apply the same reasoning to sublattices, and get Theorem 2. Propositions 10, 11, and 12 also immediately imply Theorem 3.

\section{Multiple patterns}

Theorem 1 does not generalize immediately to larger sets of patterns. In particular, even if both $S_{n}\left(\tau_{1}\right)$ and $S_{n}\left(\tau_{2}\right)$ are lattices, $S_{n}\left(\left\{\tau_{1}, \tau_{2}\right\}\right)$ is not necessarily a lattice.

Proposition 17. $S_{5}(2431,3124)$ is not a meet or join semi-lattice.

Proof. In $S_{5}$, define $\mu=43215, \mu^{\prime}=45231, \sigma=23415$, and $\sigma^{\prime}=21435$. By considering the inversion sets, we see that $\mu \wedge \mu^{\prime}=42315$ and $\sigma \vee \sigma^{\prime}=24315$. Since 42315 covers 24315 , we have that $\left\{\xi \in S_{5} \mid \xi \leqslant \mu, \xi \leqslant \mu^{\prime}, \sigma \leqslant \xi, \sigma^{\prime} \leqslant \xi\right\}=\{24315,42315\}$. Observe that $\mu, \mu^{\prime}, \sigma$, and $\sigma^{\prime}$ all avoid 2431 and 3124, but 24315 contains 2431 and 42315 contains 3124. Therefore, in the terminology of Section $3, \mu, \mu^{\prime}, \sigma$, and $\sigma^{\prime}$ form a butterfly in the Hasse diagram of $S_{5}(2431,3124)$. 
Moreover, it is not necessary for both $S_{n}\left(\tau_{1}\right)$ and $S_{n}\left(\tau_{2}\right)$ to be lattices in order for $S_{n}\left(\left\{\tau_{1}, \tau_{2}\right\}\right)$ to be a lattice. For example, consider $S_{n}(\{2134,2143\})$, which is one case of the following result.

Proposition 18. Let $T=\left\{21 \oplus \tau \mid \tau \in S_{k-2}\right\}$. Then $S_{n}(T)$ is a lattice for all $n$.

Proof. Observe that $S_{n}(T)$ is the set of permutations such that for each descent $\pi(i)>$ $\pi(i+1)$, we have $|\{j \mid j>i, \pi(j)>\pi(i)\}|<k-2$. So if $\pi \in S_{n} \backslash S_{n}(T)$, then there is a unique maximal element $\pi^{\prime} \in S_{n}(T)$ less than $\pi$. The element $\pi^{\prime}$ is obtained by changing all descents which violate the condition above to ascents. Therefore $S_{n}(T)$ is a join semi-lattice by Lemma 6 . We also have $12 \cdots n \in S_{n}(T)$, so $S_{n}(T)$ is a lattice by the dual statement of Lemma 5.

\section{Acknowledgements}

I would like to thank Nathan Reading for a conversation that inspired this project, and for comments on an earlier draft of this paper. I would also like to thank Ira Gessel for helpful conversations.

\section{References}

[1] E. Barcucci, A. Bernini, L. Ferrari, and M. Poneti, A distributive lattice structure connecting Dyck paths, noncrossing partitions and 312-avoiding permutations, Order 22 (2005), 311-328.

[2] G. Birkhoff, Lattice Theory, American Mathematical Society Colloquium Publications, vol. 25, 1948.

[3] A. Björner and F. Brenti, Combinatorics of Coxeter Groups, Graduate Texts in Mathematics, vol. 231, Springer-Verlag, New York, 2005.

[4] A. Björner and M. Wachs, Shellable nonpure complexes and posets. II, Trans. Amer. Math. Soc. 349 (1997), no. 10, 5247-5287.

[5] P. Brändén, q-Narayana numbers and the flag h-vector of $J(2 \times n)$, Discrete Mathematics 281 (2004), 67-81.

[6] S. Fomin, Duality of graded graphs, J. Alg. Combinatorics 3 (1994), 357-404.

[7] G.Th. Guilbaud and P. Rosenstiehl, Analyse algébrique d'un scrutin (French), in "Ordres totaux finis", Gauthiers-villars et Mouton, Paris, 1971, 71-100.

[8] C. Le Conte de Poly-Barbut, Sur les Trellis de Coxeter Finis (French), Math. Inf. Sci. hum. 32, no. 125 (1994), 41-57.

[9] N. Reading, Cambrian Lattices, Adv. Math. 205 (2006), no. 2, 313-353.

[10] R. Simion and F. Schmidt, Restricted Permutations, Europ. J. Combinatorics 6 (1985), 383-406. 
[11] R. Stanley, Enumerative Combinatorics, vol. 1. Cambridge University Press, Cambridge, 1999.

[12] V. Vatter, Finitely labeled generating trees and restricted permutations J. Symbolic Comput. 41 (2006), 559-572.

[13] T. Yanagimoto and M. Okamoto, Partial orderings of permutations and monotonicity of a rank correlation statistic, Ann. Inst. Statist. Math. 21 (1969), 489-506. 